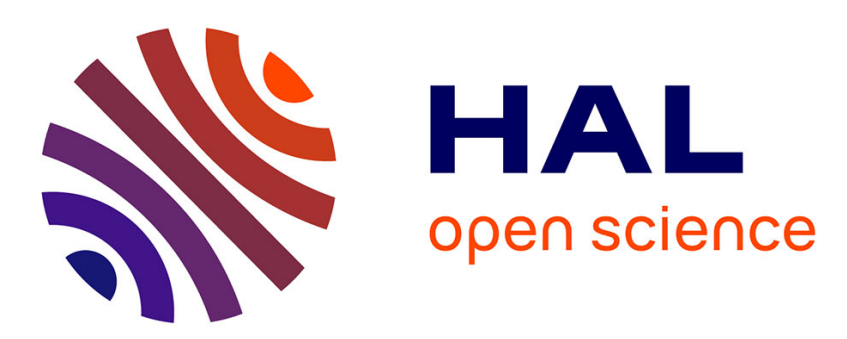

\title{
Millimeter Wave Generation Using Brillouin Scattering in a High Q Fiber Ring Resonator
}

\author{
Khaldoun Saleh, Pierre-Henri Merrer, Olivier Llopis, Gilles Cibiel
}

\section{To cite this version:}

Khaldoun Saleh, Pierre-Henri Merrer, Olivier Llopis, Gilles Cibiel. Millimeter Wave Generation Using Brillouin Scattering in a High Q Fiber Ring Resonator. International Topical Meeting on Microwave Photonics (MWP 2012), Sep 2012, Noordwijk, Netherlands. 4p. hal-00711283

\section{HAL Id: hal-00711283 \\ https://hal.science/hal-00711283}

Submitted on 22 Jun 2012

HAL is a multi-disciplinary open access archive for the deposit and dissemination of scientific research documents, whether they are published or not. The documents may come from teaching and research institutions in France or abroad, or from public or private research centers.
L'archive ouverte pluridisciplinaire HAL, est destinée au dépôt et à la diffusion de documents scientifiques de niveau recherche, publiés ou non, émanant des établissements d'enseignement et de recherche français ou étrangers, des laboratoires publics ou privés. 


\title{
Millimeter Wave Generation Using Brillouin Scattering in a High Q Fiber Ring Resonator
}

\author{
Khaldoun Saleh ${ }^{\# * 1}$, Pierre-Henri Merrer ${ }^{\# 2}$, Olivier Llopis ${ }^{\# 3}$ and Gilles Cibiel ${ }^{* 4}$ \\ ${ }^{\text {\# } C N R S ; ~ L A A S ; ~ U P S ; ~} 7$ avenue du colonel Roche, F-31077 Toulouse, France \\ CNES, 18 avenue Edouard Belin, F-31401 Toulouse, France \\ ksaleh@laas.fr
}

\begin{abstract}
We introduce an efficient technique to optically generate millimeter-wave sources. The technique is based on the frequency multiplication concept using high order harmonics, generated by a Mach-Zehnder modulator, combined with the Brillouin selective side band amplification process in a high $Q$ fiber ring resonator. We demonstrate the generation of a low phase noise $65.2 \mathrm{GHz}$ signal with a power level $15 \mathrm{~dB}$ higher than the power level usually obtained using classical frequency multiplication.
\end{abstract}

Keywords-Millimeter-wave generation; microwave photonics; fiber ring resonator; Brillouin scattering; phase noise

\section{INTRODUCTION}

Extremely high frequency generation and distribution using optical techniques represent an interesting approach. These techniques are used in many applications, from telecommunications to radio-astronomy, and at distances that vary from a few meters (e.g inside a plane or a satellite) to a few kilometers (e.g. antenna networks) and even several tens of kilometers (e.g inter-laboratories clock comparison). Many millimeter-wave ( $\mathrm{mmw}$ ) sources carried using optics have been demonstrated. Among these, the method using the beat note of a dual frequency (or mode locked) laser, where two modes are sharing the same cavity and the same gain [1], and the one based on the heterodyning of two independent lasers [2], have become quite popular, because they do not require an ultra high frequency modulator. Dual frequency lasers are particularly interesting to get high output power and high carrier to noise ratio (CNR) in the mmw range. Also, it benefits from the fact that the noise in the system affects each of the laser's two modes equally, which leads to a noise reduction in the radio frequency $(\mathrm{RF})$ domain. However, either in the case of dual mode laser or of heterodyning two independent lasers, it is difficult to get a strong correlation between the two modes, and thus an efficient rejection of the laser phase noise which degrades the beat note, and thus the resulting RF signal.

Contrarily to the above mentioned methods, frequency multiplication technique is quite easy to set up and only requires a Mach Zehnder modulator (MZM) [3]. With this approach, the optical harmonics generated by the MZM nonlinearity are strongly correlated so that the noise is equally distributed in the optical domain and thus cancelled in the RF domain. The only problem using this method is the harmonics low power levels, particularly in case of high order harmonics, which degrades the signal to noise ratio. It is thus interesting to find a way to amplify the different optical harmonics while preventing a gain competition between them. Fortunately, optical harmonics can be independently amplified thanks to the Brillouin selective sideband amplification concept (BSSA) [4].

In this paper, we present a millimeter-wave signal generation setup based on the frequency multiplication approach using a MZM to generate high optical modulation harmonics, combined with a high optical quality factor $\left(\mathrm{Q}_{\mathrm{opt}}\right)$ fiber ring resonator (FRR) in order to generate a selective Brillouin gain spectrum at each of these optical harmonics, thus independently amplifying them while maintaining a good close to the carrier phase noise of the mmw signals.

\section{FIBER RING RESONATOR}

The 20 meter long FRR used in the BSSA mm-wave signals generation (BSSA-SG) system is depicted in Fig. 1. It consists of two low loss directional couplers, linked with a single-mode polarization-maintaining (PM) optical fiber.

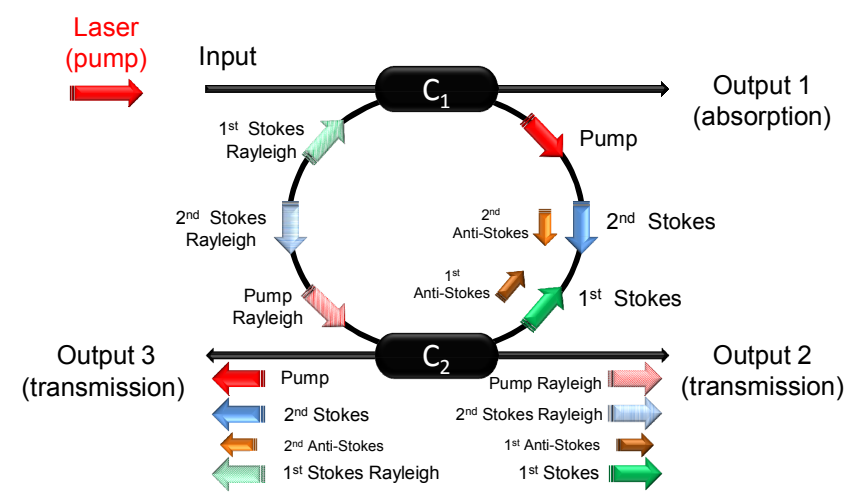

Figure 1. 20 meter long double directional coupler fiber ring resonator used to generate nonlinear optical effects (Brillouin scattering). Stokes and AntiStokes acronyms refer only to the Brillouin scattering in this scheme

Resonances occur if the total integrated phase shift of the incident laser carrier ( ${ }_{\text {laser }} \sim 1.55 \mu \mathrm{m}$ ) around the resonator ring is an integer multiple of $2 \pi \mathrm{rad}$. This resonator is thus characterized by a single transverse resonant frequency comb with a free spectral range (FSR) related to the fiber length $\mathrm{L}$ and its refractive index $\mathrm{n}: \mathrm{FSR}=\mathrm{c} /(\mathrm{n} . \mathrm{L})(\sim 10 \mathrm{MHz}$ in our case). This resonator features a $-3 \mathrm{~dB}$ bandwidth of $65 \mathrm{kHz}$, thus an optical $\mathrm{Q}_{\mathrm{opt}}$ factor of $3 \times 10^{9}$. This high $\mathrm{Q}_{\mathrm{opt}}$ factor leads

Funding from ANR project “ORA” 2010 BLAN 031203 
to a significant intra-cavity circulating power $\left(\mathrm{P}_{\text {cavity }} / \mathrm{P}_{\text {in }} \sim 54\right)$. This will reduce the thresholds of many nonlinear optical effects, especially stimulated Brillouin scattering (SBS) [5].

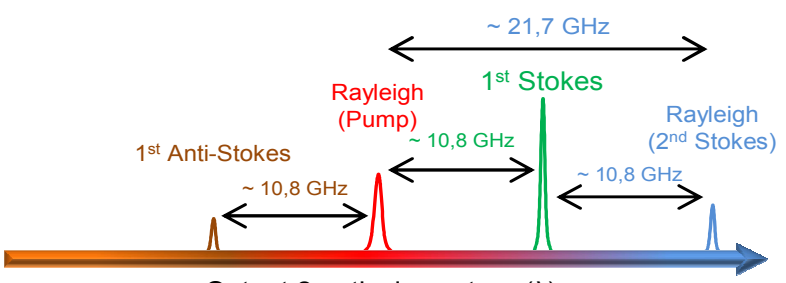

Output 2 optical spectrum $(\lambda)$

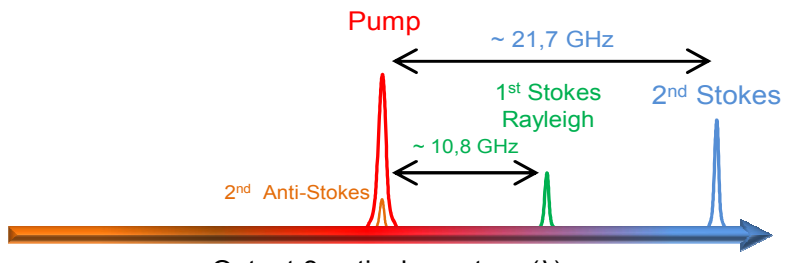

Output 3 optical spectrum $(\lambda)$

Figure 2. Theoretical optical spectrum at the second and the third outputs of the FRR, in the presence of the pump, its Rayleigh scattering and the first and second order Brillouin scattering lines and their own Rayleigh scattering lines

In optical fibers, many scattering phenomena may occur. Some of them are passive, like Fresnel reflections at the fiber end faces, and some others are active like Rayleigh backscattering, Brillouin and Raman scattering which feature two scattering modes each: spontaneous and stimulated scattering [6].

Theoretically predicted by L. Brillouin in 1922, SBS in optical fibers was firstly observed in 1964 and has been extensively studied since then [6]. It is caused by the interaction between a light wave and an acoustic wave existing already at the optical medium thermal equilibrium. At a critical incident light wave power, called stimulated Brillouin scattering threshold (SBST), the interference between the Stokes wave and the laser carrier amplifies the acoustic wave in a process called electrostriction. Thus, the scattered wave will become more intense and reinforces the interference and so on. In optical fibers, the Brillouin backscattered Stokes wave is privileged and is frequency downshifted from that of the laser carrier by $\boldsymbol{v}_{\mathrm{B}}$, set by the nonlinear medium characteristics $\left(\boldsymbol{v}_{\mathrm{B}}=10.87 \mathrm{GHz}\right.$ in our case). However, a very weak backscattered wave, called Anti-Stokes wave, and upshifted in frequency by $\boldsymbol{v}_{\mathrm{B}}$, can be also generated. When the Stokes wave power exceeds the medium SBST, it creates a second order backscattered Stokes wave downshifted in frequency from the first Stokes wave by $\boldsymbol{v}_{\mathrm{B}}$, and thus by $2 \boldsymbol{v}_{\mathrm{B}}$ from the laser carrier. The theoretical travelling directions inside the resonator and the spectral representation of the different scattering components at the resonator's second and third outputs are given in Fig. 1 and Fig. 2. The Brillouin gain bandwidth was less than $20 \mathrm{MHz}$ in the PM fibers used to fabricate the FRR, so with a resonator FSR of $10 \mathrm{MHz}$, the generated Brillouin lines inside the resonator feature a single mode operation and automatically resonate, thus taking benefit of the resonator's power enhancement factor.
Even if the generation of the Brillouin scattering is known to be very harmful to optical links and optical systems like optoelectronic oscillators [5], it can be useful in many other applications, such as low noise and ultra-narrow linewidth Brillouin lasers [7] or in modulation depth enhancement of optically carried RF signals [8]. Our aim here is to use the Brillouin effect generated at very low threshold inside the resonator, to selectively amplify the different optical modulation harmonics generated in the mmw range and thus generate high power and low phase noise $\mathrm{mmw}$ frequencies.

\section{MMW SignALS GENERATION SETUP}

The BSSA-SG system is illustrated in Fig 3. We use a Koheras laser at $1.55 \mu \mathrm{m}$ wavelength with a $1 \mathrm{kHz}$ linewidth. The optical carrier is stabilized to the FRR via a Pound-Drever -Hall loop [9], then modulated via a 20Gbits/s EOspace MZM DC biased at $\mathrm{V}_{\pi_{12}}$ in order to have a linear modulation and get high order upper and lower sidebands while keeping a high carrier power to be able to generate SBS in the FRR. Around $\mathrm{V}_{\pi / 2}$, the MZM transfer function is odd, and only odd harmonics are generated: $1,3,5 \ldots$ [3]. Then, we use the Brillouin gain generated every $10.87 \mathrm{GHz}$ multiple in the optical spectrum to amplify these harmonics.

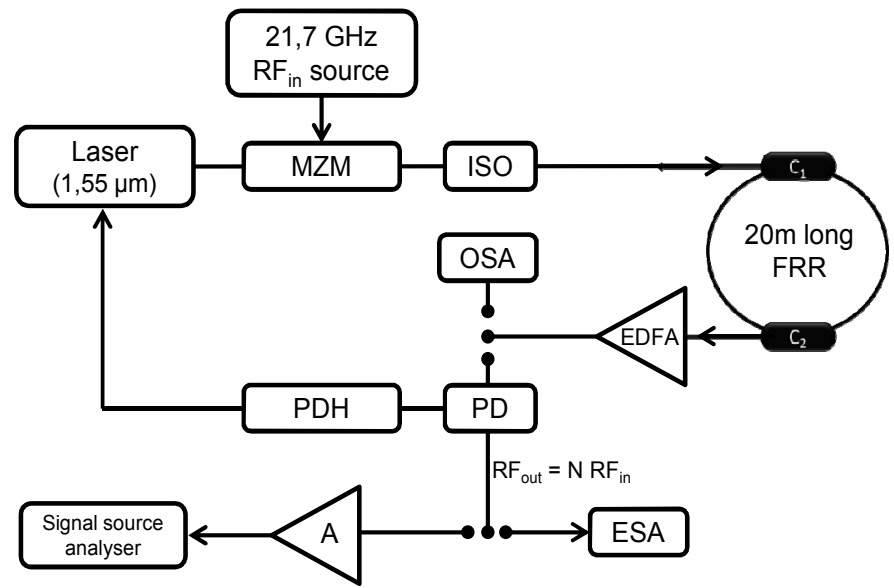

Figure 3. BSSA-SG setup using a 20 meter long FRR; ISO: isolator; EDFA: erbium doped fiber amplifier; OSA: optical spectrum analyzer; PD: photodiode; PDH: Pound-Drever-Hall laser stabilization system; ESA: electrical spectrum analyzer; A: mmw amplifier; $\cdots$. optical and RF switches

On the receiver side, we visualize the optical spectrum of the FRR third output on an Agilent HP 86142A optical spectrum analyzer (OSA) with $0.06 \mathrm{~nm}$ resolution and $\pm 1 \mathrm{~dB}$ amplitude precision, and then send it to a $75 \mathrm{GHz} \mathrm{U}^{2} \mathrm{~T}$ photodiode (PD). At the $\mathrm{PD}$ output, we visualize the RF spectrum on a $67 \mathrm{GHz}$ Rohde\&Schwarz electrical spectrum analyzer (ESA) and then analyze the signal phase noise using an Agilent E5052B signal source analyzer (SSA), which uses two equilibrated $50-85 \mathrm{GHz}$ external mixers.

\section{A. Optical Characterization}

In order to optically characterize the BSSA-SG technique, the spectrum of a $4 \mathrm{dBm}$ laser carrier at the resonator input and the spectrum of the resonator third output were visualized on the OSA. Results are depicted in Fig. 4. 


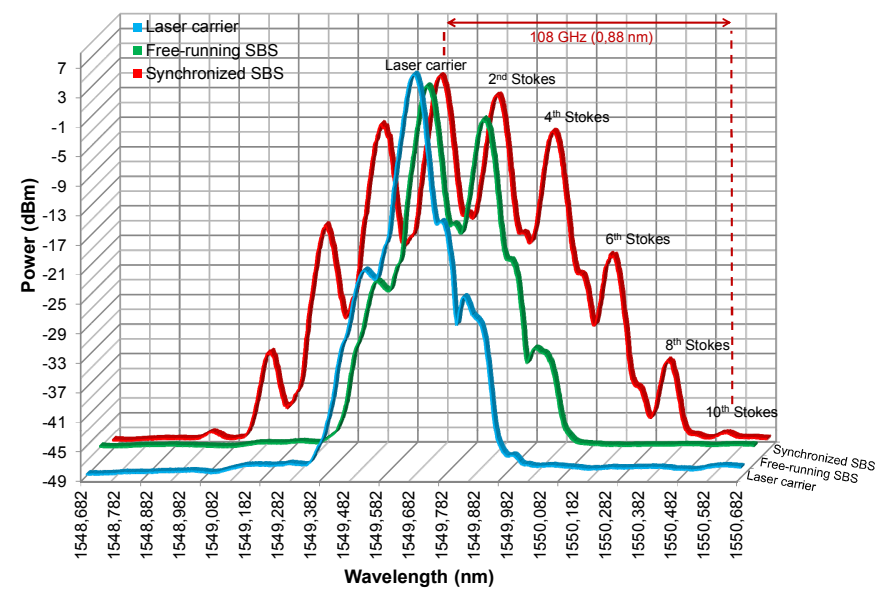

Figure 4. Optical spectrum of the laser carrier at the resonator input, of the resonator third output when Brillouin is in a free-running behavior and when modulating the laser carrier at $21.7 \mathrm{GHz}$ with a $20 \mathrm{dBm} \mathrm{RF}$ seed signal

From figure 4 , we can see that with a $4 \mathrm{dBm}$ input laser carrier, at least the second order SBS Stokes wave could be generated, spaced by $21.742 \mathrm{GHz}(0.176 \mathrm{~nm})$ from the carrier. We call it free-running SBS.

Now if a RF seed signal, having the same frequency than the second SBS line, is injected to the MZM with a high power level $(20 \mathrm{dBm})$, the modulator will produce odd upper and lower sidebands $( \pm 21.742 \mathrm{GHz}, \pm 65.226 \mathrm{GHz} \ldots)$ around the laser carrier. The higher the order of these harmonics is, the lower is their power. However, due to the Brillouin selective gain created by the carrier inside the resonator, especially Stokes lines gain, these harmonics will be amplified, and we will be able to measure even the tenth modulation harmonic, spaced by $108 \mathrm{GHz}(0.88 \mathrm{~nm})$ from the carrier, thanks to the BSSA process. Also, Brillouin scattering generation helps to increase the modulation depth [8].

\section{B. RF Characterization}

The resulting beat note of the third output on a $75 \mathrm{GHz}$ PD was observed on the ESA and results are presented in Fig. 5. The $4 \mathrm{dBm}$ input carrier leads to a high RF power free-running Brillouin scattering wave at $21.74 \mathrm{GHz}$ and a weaker one at 43.4 GHz frequency. However we can also find a $10.87 \mathrm{GHz}$ component in the RF spectrum created by the beat note of the carrier wave, the second Stokes wave and the first Stokes wave backscattered part due to the Rayleigh scattering as already mentioned in Fig. 2 .

When the laser carrier is modulated by the $21.74 \mathrm{GHz}$ seed signal with a $20 \mathrm{dBm}$ power level, the RF components at the resonator third output will be considerably amplified (odd harmonics) and we can especially notice the creation of a $65.2 \mathrm{GHz}$ component with a $-36 \mathrm{dBm}$ power level (a $6 \mathrm{~dB}$ attenuator was used at the ESA input). The $10.8 \mathrm{GHz}$, $32.6 \mathrm{GHz}$ and $54.32 \mathrm{GHz}$ even harmonics exist because of the same reason mentioned in the paragraph above, also because of the non-perfect linear modulation of the MZM.

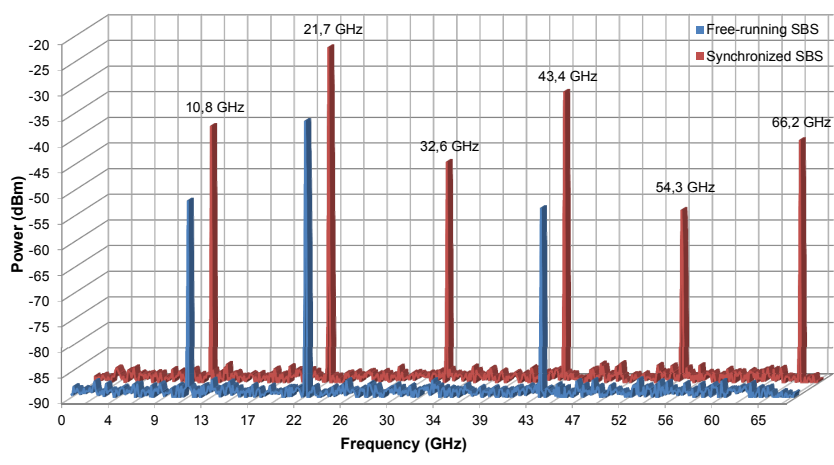

Figure 5. RF spectrum of the resonator third output when Brillouin is in a freerunning behavior and when modulating the laser carrier at $21.7 \mathrm{GHz}$ with a $20 \mathrm{dBm}$ RF seed signal. A $6 \mathrm{~dB}$ attenuator was used at the ESA input

In order to evaluate the power levels difference between the BSSA-SG method and the classical frequency multiplication method [3], the first and third harmonics power levels were measured versus the $21.7 \mathrm{GHz}$ RF seed signal power. For the classical linear modulation method, the resonator was replaced by an attenuator to simulate its intrinsic and nonlinear losses to be in the same conditions as for the BSSA-SG method. Results are presented in figure 6 .

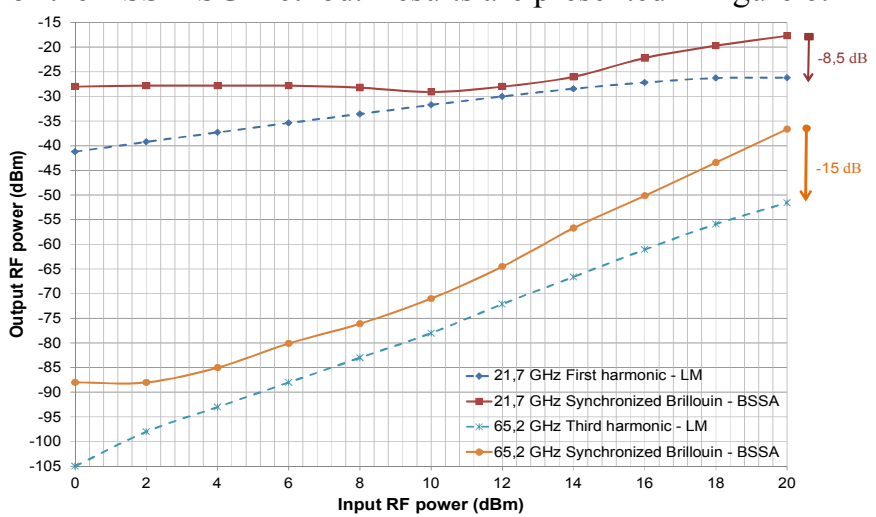

Figure 6. RF output power levels of the first and third harmonics versus the RF $21.7 \mathrm{GHz}$ seed signal power, when using the classical frequency multiplication method and when BSSA-SG technique is used

The measurements in figure 6 show clearly a real power gap between the two methods that increases with higher harmonic orders. A $15 \mathrm{~dB}$ gap at the third harmonic signal is obtained, demonstrating the gain efficiency of the BSSA-SG.

\section{Noise Characterization}

An important parameter to be characterized is the phase noise of the mmw signals generated via the BSSA-SG method. The phase noise spectrum of the Brillouin waves at $21.7 \mathrm{GHz}$ and $65.2 \mathrm{GHz}$ has been measured using the SSA. Fig. 7 shows the phase noise of the free-running Brillouin at $21.7 \mathrm{GHz}$, the synthesizer seed signal at the same frequency, and finally the phase noise of synchronized Brillouin wave via the BSSA-SG. 


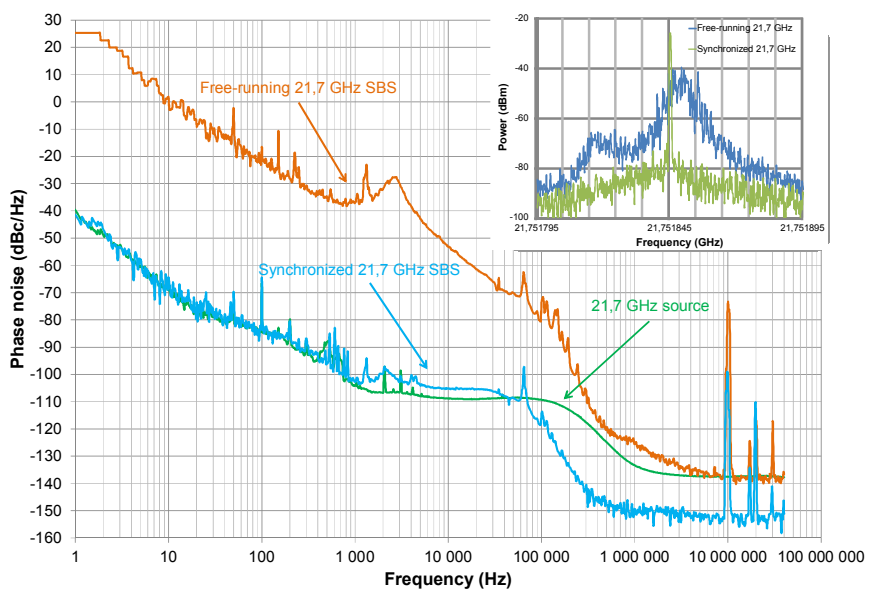

Figure 7. Phase noise of the free running Brillouin wave at $21.7 \mathrm{GHz}$, the synthesizer seed signal at the same frequency, and the synchronized Brillouin wave via the BSSA-SG method. In the upper right corner, the free-running Brillouin spectral purity enhancement was visible on the ESA after being synchronized by the seed signal (ESA: $100 \mathrm{kHz}$ span, $30 \mathrm{~Hz}$ RBW and VBW)

The impact of BSSA-SG on the spectral purity of the different free-running Brillouin components can be easily noticed even when using the ESA only, (see Fig. 7 - upper right corner). Fig. 7 results clearly demonstrate that BSSA-SG method seems to not only amplify the desired harmonics but also it significantly reduces the Brillouin gain spectrum noise, so that Brillouin gain will get almost the spectral shape and purity of the seed signal through synchronization, and thus lead to a more stable amplification.

Finally, the phase noise of the generated $65.2 \mathrm{GHz}$ signal has been measured and compared to the original $21.7 \mathrm{GHz}$ seed signal phase noise and to its theoretical phase noise after being multiplied by $3,(+20 \log (\mathrm{N})$ where $\mathrm{N}$ is the frequency multiplication factor), see Fig. 8.

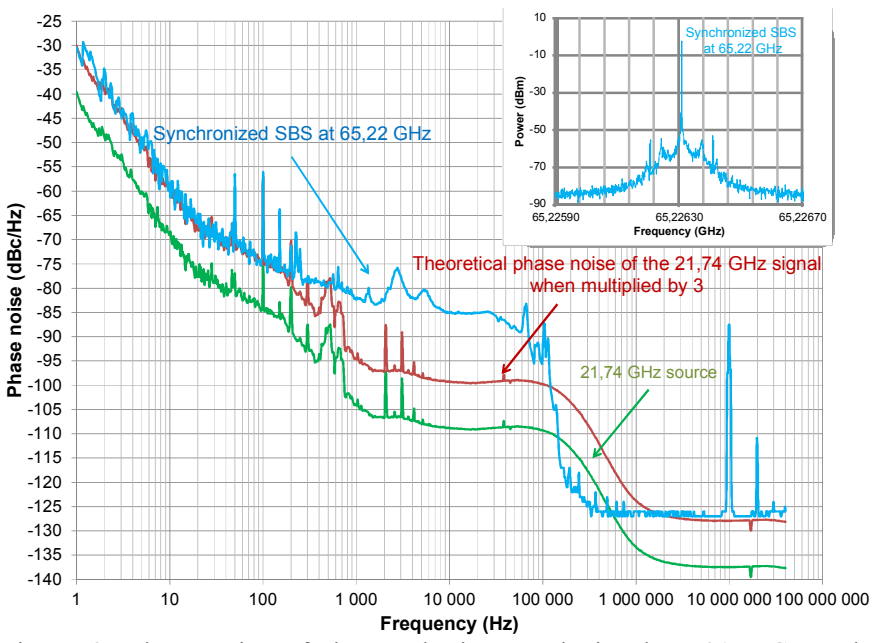

Figure 8. Phase noise of the synthesizer seed signal at $21.7 \mathrm{GHz}$, the synthesizer phase noise multiplied to $65.2 \mathrm{GHz}$ and the generated $65.2 \mathrm{GHz}$ signal via BSSA-SG method

We can notice that the synchronized signal at $65.2 \mathrm{GHz}$ features the same phase noise spectrum as if it was generated by the classical multiplication method, at least in the close to carrier noise range from $1 \mathrm{~Hz}$ till $600 \mathrm{~Hz}$ offset frequencies. The excess noise at frequencies from $600 \mathrm{~Hz}$ to $80 \mathrm{kHz}$ seems to be generated by the PDH loop noise, the Rayleigh scattering noise of the different optical spectrum components and the Brillouin gain noise. The rapid decrease in the phase noise after the $40 \mathrm{kHz}$ offset frequency is due to the optical resonator filtering effect. The PDH noise part can be reduced if a double PDH stabilization technique controlling the laser and the resonator at the same time is used, like in [10] were interesting $\mathrm{mmw}$ signals generation system stability and accurate tunability were demonstrated. However, high order mmw signals generation with low phase noise is possible with our BSSA-SG method, where there is theoretically no limit in the generation of higher order Brillouin lines; The only limit will be at the receiver part, especially the photodiode bandwidth.

\section{CONCLUSION}

Investigations have been performed on the generation of millimeter-wave signals using Brillouin selective sideband amplification process in a high $\mathrm{Q}$ fiber ring resonator. The synchronization of Brillouin lines has resulted in high spectral purity millimeter-wave signals generation up to $65 \mathrm{GHz}$, which is of great interest for many applications. The proposed technique allows a high signal to noise ratio and a low close to carrier phase noise.

\section{REFERENCES}

[1] G. Pillet, L. Morvan, M. Brunel, F. Bretenaker, D. Dolfi, M. Vallet, J.P. Huignard, and A.L. Floch, "Dual-frequency laser at $1.5 \mathrm{~lm}$ for optical distribution and generation of high-purity microwave signals," J. Lightwave Technol., vol. 26, issue 15, pp. 2764-2773, August 2008.

[2] G. J. Simonis, and D. G. Purchase, "Optical generation, distribution, and control of microwaves using laser heterodyne," IEEE Trans. Microw. Theory Tech., vol. 38, issu 5, pp. 667-669, May 1990.

[3] O. Llopis , P. Martinet, P. H. Merrer, and K. Saleh, "50-60 GHz local oscillator transmission over fiber using optical frequency multiplication," Microw. Opt. Technol. Lett., vol. 54, issu 5, pp. 13131316, May 2012.

[4] X.S. Yao, "Brillouin selective sideband amplification of microwave photonic signals," Photonics Technology Letters, IEEE, vol.10, no.1, pp.138-140, Jan. 1998.

[5] K. Saleh, P.H. Merrer, O. Llopis, and G. Cibiel, "Optical scattering noise in high Q fiber ring resonators and its effect on optoelectronic oscillator phase noise," Opt. Lett., vol. 37, pp. 518-520, Feb. 2012.

[6] G.P. Agrawal, "Nonlinear Fiber Optics," 4th ed. Academic Press, 2007.

[7] G. Jihong, S. Staines, W. Zuolan, Z. Jie, M. Blake and J. Shibin, "Highly stable low-noise Brillouin fiber laser with ultranarrow spectral linewidth," IEEE Photonics Technology Letters, vol. 18, no.17, pp.18131815, Sept. 2006.

[8] S. Norcia, S. Tonda-Goldstein, D. Dolfi, J.-P. Huignard, and R. Frey, "Efficient single-mode Brillouin fiber laser for low-noise optical carrier reduction of microwave signals," Opt. Lett. , vol. 28, issue 20, pp. 18881890, Oct. 2003.

[9] R. W. P. Drever, J. L. Hall, F. V. Kowalski, J. Hough, G. M. Ford, A. J. Munley, and H. Ward, "Laser phase and frequency stabilization using an optical resonator,” Appl. Phys. B, vol. 31, pp. 97-105, 1983.

[10] M. C. Gross, P. T. Callahan, T. R. Clark, D. Novak, R. B. Waterhouse, and M. L. Dennis, "Tunable millimeter-wave frequency synthesis up to $100 \mathrm{GHz}$ by dual-wavelength Brillouin fiber laser," Opt. Express, vol 18 , no. 13 , pp. 13321-13330, June 2010. 